\title{
Stability and Controllability of a Class of 2-D Linear Systems With Dynamic Boundary Conditions
}

\author{
E. Rogers, K. Galkowski, A. Gramacki, J. Gramacki, and D. H. Owens
}

\begin{abstract}
Discrete linear repetitive processes are a distinct class of two-dimensional (2-D) linear systems with applications in areas ranging from long-wall coal cutting through to iterative learning control schemes. The feature which makes them distinct from other classes of 2-D linear systems is that information propagation in one of the two independent directions only occurs over a finite duration. This, in turn, means that a distinct systems theory must be developed for them. In this paper a complete characterization of stability and so-called pass controllability (and several resulting features), essential building blocks for a rigorous systems theory, under a general set of initial, or boundary, conditions is developed. Finally, some significant new results on the problem of stabilization by choice of the pass state initial vector sequence are developed.
\end{abstract}

Index Terms-Controllability, dynamic boundary conditions, repititive processes, stability, stabilization.

\section{INTRODUCTION}

$\mathbf{T}$ HE essential unique characteristic of a repetitive process (also termed a multipass process in the early literature) can be illustrated by considering machining operations where the material or workpiece involved is processed by a series of sweeps, or passes, of the processing tool. Assuming the pass length $\alpha<+\infty$ to be constant, the output vector, or pass profile, $y_{k}(t), 0 \leq t \leq \alpha,(t$ being the independent spatial or temporal variable), generated on the $k$ th pass acts as a forcing function on, and hence contributes to, the dynamics of the new pass profile $y_{k+1}(t), 0 \leq t \leq \alpha, k \geq 0$.

Industrial examples of repetitive processes include long-wall coal cutting operations and metal rolling operations [4], [11]. Also a number of so-called algorithmic examples exist where adopting a repetitive process setting for analysis has clear advantages over alternative approaches to systems related analysis. These include iterative learning control schemes [1], [2] and iterative solution algorithms for dynamic nonlinear optimal control problems based on the maximum principle [8].

The essential unique control problem for these processes is that the output sequence of pass profiles generated can contain oscillations that increase in amplitude in the pass to pass

Manuscript received April 3, 2000; revised April 25, 2001. This paper was recommended by Associate Editor S. Basu.

E. Rogers is with the Department of Electronics and Computer Science, University of Southampton, Southampton SO17 1BJ, U.K. (e-mail: etar@ecs.soton.ac.uk).

K. Galkowski is with the Institute of Control and Computation Engineering, University of Zielona Gora, Poland.

A. Gramacki and J. Gramacki are with the Institute of Informatics and Electronics, University of Zielona Gora, Poland.

D. H. Owens is with the Department of Automatic Control and Systems Engineering, University of Sheffield, Sheffield S1 3JD, U.K.

Publisher Item Identifier S 1057-7122(02)01186-8. (i.e., $k$ ) direction. Such behavior is easily generated in simulation studies and in experiments on scaled models of industrial processes such as long-wall coal cutting. In this last case, these oscillations are caused by the machine's weight as it comes to rest on the newly cut floor profile ready for the start of the next pass of the coal face.

Attempts to remove this problem by standard, or one-dimensional (1-D), control action fail precisely because such an approach ignores their inherent two-dimensional (2-D) systems structure, i.e., information propagation along a given pass $(t$ direction) and from pass to pass ( $k$ direction). In particular, so-called discrete linear repetitive processes considered in this paper are a distinct class of 2-D discrete linear systems where, in contrast to 2-D discrete linear systems described by the well known and extensively studied Roesser [9] and Fornasini Marchesini [5] models, information propagation in one of the two independent directions only occurs over a finite duration.

The 1-D approach to stability analysis for these processes completely ignores the effects of resetting one part of the initial, or boundary, conditions before the start of each new pass. This is a key distinguishing feature of these processes and there is evidence to strongly suggest that the form of the boundary conditions, and, in particular, the pass state initial vector sequence in the defining state-space model, at the start of each new pass is critical to their stability and control properties [11].

In this paper, a general set of boundary conditions for discrete linear repetitive processes is defined and a complete characterization of stability under these conditions is obtained. Also a previously developed 1-D equivalent discrete linear systems state-space model of the underlying dynamics is used to develop new results which lead to computationally feasible stability tests and the characterization of physically relevant controllability properties. Finally, the problem of stabilization by choice of the pass state initial vector sequence is solved. The stability analysis starts from the stability theory for linear repetitive processes developed in [10] on the basis of an abstract model in a Banach space setting which includes all such processes as special cases and therefore a fundamental task is to interpret the resulting abstract model stability conditions to the particular case (or sub-class) under consideration, which is where new stability results in this paper arise.

\section{BACKGROUND}

The state-space model of a discrete linear repetitive process has the following form over $0 \leq p \leq \alpha-1, k \geq 0$, where on pass $k, x_{k}(p)$ is the $n \times 1$ state vector, $y_{k}(p)$ is the $m \times 1$ vector 
pass profile, and $u_{k}(p)$ is the $r \times 1$ vector of control inputs (and $k, p, \alpha \in \mathbb{N})$

$$
\begin{aligned}
x_{k+1}(p+1) & =A x_{k+1}(p)+B u_{k+1}(p)+B_{0} y_{k}(p) \\
y_{k+1}(p) & =C x_{k+1}(p)+D u_{k+1}(p)+D_{0} y_{k}(p) .
\end{aligned}
$$

To complete the process description, it is necessary to specify the 'initial conditions' - termed the boundary conditions here, i.e., the state initial vector on each pass and the initial pass profile. The simplest possible form for these is

$$
\begin{aligned}
x_{k+1}(0) & =d_{k+1}, k \geq 0 \\
y_{0}(p) & =y(p), 0 \leq p \leq \alpha-1
\end{aligned}
$$

where $d_{k+1}$ is an $\mathrm{n} \times 1$ vector with constant entries and $y(p)$ is an $\mathrm{m} \times 1$ vector whose entries are known functions of $p$. These boundary conditions occur in the iterative learning control application where the repetitive processing setting provides distinct advantages over alternatives for the case of linear dynamics. In particular, studying the stability of such schemes in a repetitive process setting also produces, for no extra computational cost, precise information on stability and, uniquely, on both the rate of convergence to the learned signal and the errors generated on successive passes (termed trials in the iterative learning control literature).

In some cases, the boundary conditions of (2) are simply not strong enough to 'adequately' model the underlying dynamics-even for preliminary simulation/control analysis. For example, the optimal control application [8] requires the use of pass state initial vectors which are a function of the previous pass profile. In this and the next section, we define a general set of boundary conditions for discrete linear repetitive processes and obtain a complete characterization of stability under these conditions.

Clearly, it is of prime importance to start with the general form of boundary conditions with subsequent specialization to particular cases as required. Other work [11] has concluded that the most general set results from replacing $x_{k+1}(0), k \geq 0$, in (2) by

$$
x_{k+1}(0)=d_{k+1}+\sum_{j=0}^{\alpha-1} K_{j} y_{k}(j)
$$

where $K_{j}, 0 \leq j \leq \alpha-1$, are $\mathrm{n} \times \mathrm{m}$ matrices with constant entries. These are precisely of the form required in the optimal control application and the existence of this application (where the major interest is in the convergence properties of the iterative solution algorithm) provides a major motivation to undertake a detailed investigation of the systems theoretic properties of processes described by (1) and (3), and for which the results in this paper are the first major outcomes of such an investigation. Fig. 1 illustrates the boundary conditions for the discrete linear repetitive processes under consideration here.

The stability theory for linear constant pass length repetitive processes is based on the following abstract model of the underlying dynamics where $E_{\alpha}$ is a suitably chosen Banach space with norm $\|$.$\| and W_{\alpha}$ is a linear subspace of $E_{\alpha}$

$$
y_{k+1}=L_{\alpha} y_{k}+b_{k+1}, k \geq 0 \text {. }
$$

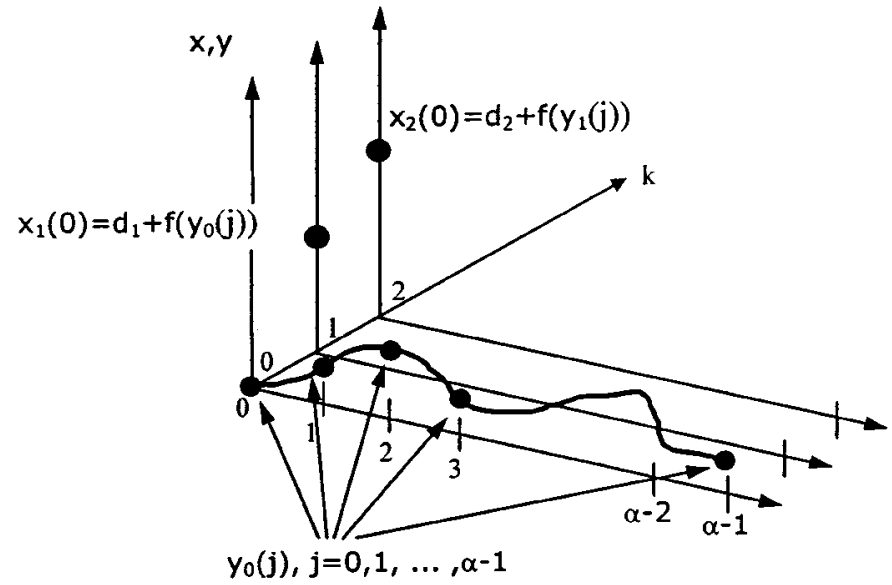

Fig. 1. Illustrating the dynamic boundary conditions.

In this model, $y_{k} \in E_{\alpha}$ is the pass profile on pass $k, b_{k+1} \in W_{\alpha}$, and $L_{\alpha}$ is a bounded linear operator mapping $E_{\alpha}$ into itself. The term $L_{\alpha} y_{k}$ represents the contribution from pass $k$ to pass $k+1$ and $b_{k+1}$ represents known initial conditions, disturbances and control input effects. We denote this model by $S$.

At this stage, introduce the Banach space $E_{\alpha}=\ell_{2}^{m}[0, \alpha]$ of real $m \times 1$ vectors (corresponding to $p=0,1,2, \ldots, \alpha-1$, in (1)). Also regard $y_{k}=\left[y_{k}(0), y_{k}(1), \ldots, y_{k}(\alpha-1)\right]$ as a point in $E_{\alpha}$. Then it is routine to show that (1) and (3) define a special case of (4) with

$$
\begin{aligned}
\left(L_{\alpha} y_{k}\right)(p)=C A^{p} \hat{y}+\sum_{r=0}^{p-1} C A^{p-1-r} B_{0} y_{k}(r) & \\
& +D_{0} y_{k}(p), 0 \leq p \leq \alpha-1
\end{aligned}
$$

where

$$
\hat{y}=\sum_{j=0}^{\alpha-1} K_{j} y_{k}(j)
$$

and

$$
\begin{aligned}
b_{k+1}(p)=C A^{p} d_{k+1}+\sum_{r=0}^{p-1} C A^{p-1-r} B u_{k+1}(r) \\
+D u_{k+1}(p), 0 \leq p \leq \alpha-1 .
\end{aligned}
$$

\section{STABILITY ANALYSIS}

The linear repetitive process $S$ is said to be asymptotically stable [10] if $\exists$ a real scalar $\delta>0$ such that, given any initial profile $y_{0}$ and any disturbance sequence $\left\{b_{k}\right\}_{k \geq 1} \in W_{\alpha}$ bounded in norm (i.e., $\left\|b_{k}\right\| \leq c_{1}$ for some constant $c_{1} \geq 0$ and $\forall k \geq 1$ ), the output sequence generated by the perturbed process

$$
y_{k+1}=\left(L_{\alpha}+\gamma\right) y_{k}+b_{k+1}, k \geq 0
$$

is bounded in norm whenever $\|\gamma\| \leq \delta$.

This definition is easily shown to be equivalent to the requirement that $\exists$ finite real scalars $M_{\alpha}>0$ and $\lambda_{\alpha} \in(0,1)$ such that

$$
\left\|L_{\alpha}^{k}\right\| \leq M_{\alpha} \lambda_{\alpha}^{k}, k \geq 0
$$

(where $\|$.$\| is also used to denote the induced operator norm).$ 
A necessary and sufficient condition [10] for (9) to hold is that the spectral radius, $r\left(L_{\alpha}\right)$, of $L_{\alpha}$ satisfies

$$
r\left(L_{\alpha}\right)<1 \text {. }
$$

Consider also the case when $x_{k+1}(0)$ is of the form given in (2). Then, in this case, it is known that the necessary and sufficient condition for asymptotic stability is that $r\left(D_{0}\right)<1$. This is clearly a necessary condition for asymptotic stability under the pass state initial conditions of (3) and is hence assumed to hold in what follows. Also, it is convenient to first establish the condition for asymptotic stability in the special case when $D_{0}=0$.

Introduce

$$
M(\zeta):=\sum_{j=0}^{\alpha-1} K_{j} C\left(A+\zeta^{-1} B_{0} C\right)^{j}
$$

where $\zeta \neq 0$ is a complex scalar. Then, we have the following result.

Theorem: Suppose that $\left\{A, B_{0}\right\}$ is controllable, and consider the linear repetitive process $S$ generated by (1) and (3) (with $D_{0}=0$ ). Then the resulting bounded linear operator $L_{\alpha}$ defined, in this case, by (5) and (6) has spectral radius given by

$$
\left.r\left(L_{\alpha}\right)=\sup \left\{|\zeta|: \operatorname{det}\left(\zeta I_{n}-M(\zeta)\right)=0\right\}\right\}
$$

Proof: Evaluation of $r\left(L_{\alpha}\right)$ for any case is equivalent to finding the spectral value (or eigenvalue in the finite dimensional case) of this bounded linear operator which has the largest modulus. To do this, we follow a standard route in functional analysis (see, for example, [10] and the relevant cited references) and examine the problem of solving the equation ( $\zeta I-$ $\left.L_{\alpha}\right) y=f$ for $y \in E_{\alpha}$ when $f \in E_{\alpha}$. In particular, we determine the set of all values of $\zeta$ such that the map $f \mapsto y$ is defined and bounded in $E_{\alpha}$. Then by definition $r\left(L_{\alpha}\right)$ is obtained by taking the supremum of the absolute values of the members of this set (in effect, the member of this set with the largest modulus).

Write $\eta=L_{\alpha} y$. Then $\zeta y-\eta=f \Rightarrow y=\zeta^{-1}(f+\eta)$ and the relationship between $f$ and $\eta$ is described by

$$
\begin{aligned}
x(p+1) & =A x(p)+B_{0} y(p) \\
\eta(p) & =C x(p), x(0)=\sum_{j=0}^{\alpha-1} K_{j} y(j) .
\end{aligned}
$$

Using $y=\zeta^{-1}(f+\eta)$ we now have

$$
\begin{aligned}
x(p+1) & =\left(A+\zeta^{-1} B_{0} C\right) x(p)+\zeta^{-1} B_{0} f(p) \\
y(p) & =\zeta^{-1}(C x(p)+f(p))
\end{aligned}
$$

and the general solution for $x(p)$ can be written in the form

$$
\begin{aligned}
x(p)=\left(A+\zeta^{-1} B_{0} C\right)^{p} \sum_{j=0}^{\alpha-1} \zeta^{-1} K_{j}[C x(j)+f(j)] & \\
& +\sum_{j=0}^{p-1} \zeta^{-1}\left(A+\zeta^{-1} B_{0} C\right)^{p-1-j} B_{0} f(j) .
\end{aligned}
$$

The existence of a solution $y$ to $\left(\zeta I-L_{\alpha}\right) y=f$ is equivalent to the consistency of (15) at the points $p=0,1,2, \cdots, \alpha-1$. Also, the $\alpha$ equations obtained by successively evaluating the equation for $x(p)$ given by (15) at $p=0,1,2, \cdots, \alpha-1$, can be written in matrix form as $\left[\zeta I_{n \alpha}-\hat{M}(\zeta)\right] X=g(\zeta) f$, where the map $f \mapsto g(\zeta) f$ is bounded and $X=\left[x^{T}(0), \cdots, x^{T}(\alpha-1)\right]^{T}$, and entry $(h, v)$ in the $n \alpha \times n \alpha$ matrix $\hat{M}(\zeta)$ is the $n \times n$ matrix $\left(A+\zeta^{-1} B_{0} C\right)^{h} K_{v} C$. It is now routine to show that a sufficient condition for the existence of a solution $X$ is that $\left(\zeta I_{n \alpha}-\hat{M}(\zeta)\right) \neq 0$.

In this situation, it is a standard fact that $\zeta$ is not a spectral value of $L_{\alpha}$, i.e., $\zeta \notin \sigma\left(L_{\alpha}\right)$ where $\sigma(\cdot)$ denotes the spectrum of its argument. Also it is easy to conclude that this nonsingularity condition is necessary by using the controllability assumption to construct an $f$ such that there is no solution to $\left[\zeta I_{n \alpha}-\right.$ $\hat{M}(\zeta)] X=f(\zeta)$, and hence no solution to $\left(\zeta I-L_{\alpha}\right) y=f$ for that choice of $f$. Finally, it is easy to see that the matrix $\hat{M}(\zeta)$ can be written as

$$
\hat{M}(z)=\left[\begin{array}{c}
I_{n} \\
\vdots \\
\left(A+\zeta^{-1} B_{0} C\right)^{\alpha-1}
\end{array}\right]\left[K_{0} C, \cdots, K_{\alpha-1} C\right]
$$

and note that $\operatorname{det}\left(\zeta I_{n \alpha}-\hat{M}(\zeta)\right)=\zeta^{n(\alpha-1)} \operatorname{det}\left(\zeta I_{n}-\right.$ $\left.\sum_{j=0}^{\alpha-1} K_{j} C\left(A+\zeta^{-1} B_{0} C\right)^{j}\right)$. Hence

$$
\sigma\left(L_{\alpha}\right)=\left\{\zeta: \operatorname{det}\left(\zeta I_{n}-\sum_{j=0}^{\alpha-1} K_{j} C\left(A+\zeta^{-1} B_{0} C\right)^{j}\right)=0\right\} .
$$

Corollary 1: The linear repetitive process $S$ generated by (1) and (3) (with $D_{0}=0$ ) is asymptotically stable if, and only if, all solutions of

$$
\operatorname{det}\left(\zeta I_{n}-\sum_{j=0}^{\alpha-1} K_{j} C\left(A+\zeta^{-1} B_{0} C\right)^{j}\right)=0
$$

have modulus strictly less than unity.

Further simplification (reduction in dimension) is possible in some cases, e.g., the following.

Corollary 2: Consider the linear repetitive process $S$ generated by (1) and (3) (with $D_{0}=0$ ) in the special case when $K_{j}=K T_{j}, 0 \leq j \leq \alpha-1$, where $K$ is an $n \times m$ matrix with constant entries and $T_{j}, 0 \leq j \leq \alpha-1$, is an $m \times m$ matrix with constant entries. Then this process is stable along the pass if, and only if, all solutions of

$$
\operatorname{det}\left(\zeta I_{m}-\sum_{j=0}^{\alpha-1} T_{j} C\left(A+\zeta^{-1} B_{0} C\right)^{j} K\right)=0
$$

have modulus strictly less than unity.

Note: Often, it will be the case that $n \gg m$, i.e., the pass state vector dimension is much greater than that of the pass profile vector and, where it applies, this last corollary offers great computational savings.

In the case when $D_{0} \neq 0$, first define the following transformation:

$$
v_{k+1}(p):=y_{k+1}(p)-D_{0} y_{k}(p), 0 \leq p \leq \alpha-1, k \geq 0 .
$$

Then, it is routine that the proof Theorem 1 still holds under the following substitutions

$$
\begin{aligned}
& B_{0} \rightarrow \zeta B_{0}\left(\zeta I_{m}-D_{0}\right)^{-1} \\
& K_{j} \rightarrow \zeta K_{j}\left(\zeta I_{m}-D_{0}\right)^{-1} .
\end{aligned}
$$

Hence we have the following result. 
Corollary 3: The linear repetitive process $S$ generated by (1) and (3) with $r\left(D_{0}\right)<1$ is asymptotically stable if, and only if, all solutions of

$$
\begin{aligned}
\operatorname{det}\left(\zeta I_{n}-\sum_{j=0}^{\alpha-1} K_{j} \zeta\left(\zeta I_{m}-D_{0}\right)^{-1}\right. & \\
& \left.\times C\left(A+B_{0}\left(\zeta I_{m}-D_{0}\right)^{-1} C\right)^{j}\right)=0
\end{aligned}
$$

have modulus strictly less than unity.

Consider now the case when the boundary conditions are of the simple form (2). Then we have the 'counter-intuitive' result that asymptotic stability is essentially independent of the process dynamics and, in particular, the eigenvalues of the matrix $A$. This is due entirely to the fact that the pass length $\alpha$ is finite and of constant value for all passes. This situation will change drastically if (as below) we let $\alpha \rightarrow \infty$.

In general, Theorem 1 shows that the property of asymptotic stability for discrete linear repetitive processes is critically dependent on the structure of $x_{k+1}(0), k \geq 0$. Suppose also that this sequence is incorrectly modeled as in (2) instead of a special case of (3). Then, the process could well be interpreted as asymptotically stable when in actual fact it is asymptotically unstable!

The above analysis provides necessary and sufficient conditions for asymptotic stability but no really 'useful' information concerning transient behavior and, in particular, about the behavior of the output sequence of pass profiles as the process evolves from pass to pass (i.e., in the $k$ direction). The limit profile provides a characterization of process behavior after a 'large number' of passes has elapsed.

Suppose that the abstract model $S$ is asymptotically stable and let $\left\{b_{k}\right\}_{k \geq 1}$ be a disturbance sequence that converges strongly to a disturbance $b_{\infty}$. Then the strong limit

$$
y_{\infty}:=\lim _{k \rightarrow+\infty} y_{k}
$$

is termed the limit profile corresponding to this disturbance sequence. Also it can be shown that $y_{\infty}$ is uniquely given by

$$
y_{\infty}=\left(I-L_{\alpha}\right)^{-1} b_{\infty} .
$$

Note also that (24) can be formally obtained from (4) (which describes the dynamics of $S$ ) by replacing all variables by their strong limits,i.e., $y_{k+1}$ and $y_{k}$ by $y_{\infty}$ and $b_{k+1}$ by $b_{\infty}$ and noting that asymptotic stability ensures that $I-L_{\alpha}$ is invertible.

Proposition 1: In the case when $S$ generated by (1) and (3) is asymptotically stable, the resulting limit profile is

$$
\begin{aligned}
x_{\infty}(p+1)= & \left(A+B_{0}\left(I_{m}-D_{0}\right)^{-1} C\right) x_{\infty}(p) \\
& +\left(B+B_{0}\left(I_{m}-D_{0}\right)^{-1} D\right) u_{\infty}(p) \\
y_{\infty}(p)= & \left(I_{m}-D_{0}\right)^{-1} C x_{\infty}(p) \\
& +\left(I_{m}-D_{0}\right)^{-1} D u_{\infty}(p) \\
x_{\infty}(0)= & \left(I_{n}-\left(\sum_{j=0}^{\alpha-1} K_{j}\left(I_{m}-D_{0}\right)^{-1} C(A\right.\right. \\
& \left.\left.\left.+B_{0}\left(I_{m}-D_{0}\right)^{-1} C\right)^{j}\right)\right)^{-1} d_{\infty}
\end{aligned}
$$

where $d_{\infty}$ is the strong limit of $\left\{d_{k}\right\}_{k \geq 1}$.
Proof: Replace all variables by their strong limits and note that, by considering the unforced case, we obtain the form of $x_{\infty}(0)$ as the required inverses exist by asymptotic stability.

Asymptotic stability of processes described by (1) and (3) guarantees the existence of a limit profile which is described by a standard, or 1-D, discrete linear systems state-space model. Hence, in effect, if the process under consideration is asymptotically stable, then its repetitive dynamics can, after a 'sufficiently large' number of passes, be replaced by those of a 1-D discrete linear time invariant system. This result has obvious implications in terms of the design of control schemes for these processes.

Owing to the finite pass length (over which duration even an unstable 1-D linear system can only produce a bounded output), asymptotic stability cannot guarantee that the resulting limit profile has 'acceptable' along the pass dynamics, where in this case the basic requirement is stability as a 1-D linear system. As a simple example to demonstrate this fact, consider the case when $A=-0.5, B=1, B_{0}=0.5+\beta, C=1, D=0 . D_{0}=0$, $x_{k+1}(0)=0, k \geq 0$, where $\beta$ is a real scalar. Then in the case when $|\beta| \geq 1$, the resulting limit profile dynamics are described by the unstable 1-D linear system

$$
y_{\infty}(p+1)=\beta y_{\infty}(p)+u_{\infty}(p) .
$$

The natural definition of 'stability along the pass' for the above example is to ask that the limit profile is stable in the sense that $|\beta|<1$ if we let the pass length $\alpha$ become infinite. This intuitively appealing idea is, however, not applicable to cases where the limit profile resulting from asymptotic stability is not described by a 1-D linear systems state-space model. Consequently stability along the pass for the general model $S$ has been defined in terms of the rate of approach to the limit profile as the pass length $\alpha$ becomes infinitely large. One of several equivalent formulations of this property is that $S$ is said to be stable along the pass if, and only if, $\exists$ real numbers $M_{\infty}>0$ and $\lambda_{\infty} \in(0,1)$ which are independent of $\alpha$ and satisfy

$$
\left\|L_{\alpha}^{k}\right\| \leq M_{\infty} \lambda_{\infty}^{k}, \forall \alpha>0, \forall k \geq 0 .
$$

In physical terms, the essential difference between asymptotic stability and stability along the pass can be highlighted by noting that they are, in effect, formulated in bounded-input bounded-output (BIBO) terms, where the term 'bounded' is defined in terms of the norm on the underlying function space. The concept of asymptotic stability demands that sequences of inputs (i.e., in terms of the abstract model $\left\{b_{k}\right\}_{k \geq 1}$ ) produce bounded pass profile sequences $\left(\left\{y_{k}\right\}_{k \geq 1}\right)$ over the finite pass length. Stability along the pass, however, is stronger in the sense that it demands this property uniformly with respect to the pass length.

Necessary and sufficient conditions [10] for (27) are that

$$
r_{\infty}:=\sup _{\alpha>0} r\left(L_{\alpha}\right)<1
$$

and that

$$
M_{0}:=\sup _{\alpha>0} \sup _{|\zeta| \geq \lambda}\left\|\left(\zeta I-L_{\alpha}\right)^{-1}\right\|<\infty
$$

for some real number $\lambda \in\left(r_{\infty}, 1\right)$. Note also that (29) here does, in fact, imply (28). The reason for retaining their separate iden- 
tities is that in many of the cases considered to date, including the one here (see below), (28) has proved much easier to interpret than (29). Also as it is a necessary condition, if (28) does not hold for a particular case then there is no need to consider (29).

In the case of (28) for processes described by (1) and (3), first note that it follows immediately from the proof of Theorem 1 that the spectrum of $L_{\alpha}$ in this case is independent of the points on the previous pass profile defined by the index $j$ in (3). Hence, (28) for stability along the pass in this case holds if, and only if, Corollary 3 holds.

To examine the 'boundedness' condition of (29), the method is to note that this condition is equivalent to the existence of a $\lambda \in\left(r_{\infty}, 1\right)$ such that the equation $\left(\zeta I-L_{\alpha}\right) y=f$ in $E_{\alpha}$ has a uniformly bounded, with respect to $\alpha$, solution $y$ for all choices of $f \in E_{\infty}$ satisfying $\sup _{\alpha>0}\|f\|<\infty$ and $\forall|\zeta| \geq 1$. Clearly, a necessary condition for this property is that $r(A)<1$ (Equivalently, the first pass profile produced is uniformly bounded with respect to $\alpha$.) This condition is taken as an assumption in the following result which gives necessary and sufficient conditions for stability along the pass.

Theorem 2: Suppose that $\left\{A, B_{0}\right\}$ is controllable, $\{C, A\}$ is observable, $r\left(D_{0}\right)<1$, and $r(A)<1$. Then the linear repetitive process $S$ generated by (1) and (3) is stable along the pass if, and only if

1) Corollary 3 holds;

2) all eigenvalues of the transfer function matrix.

$$
G(\rho)=C\left(\rho I_{n}-A\right)^{-1} B_{0}+D_{0}
$$

have modulus strictly less than unity $\forall|\rho|=1$.

Proof: The necessity of (a) follows as $r\left(L_{\alpha}\right) \leq r_{\infty}<1$ requires asymptotic stability on finite intervals. To prove the necessity of (b), it is necessary to consider unbounded intervals. Write $[0,+\infty)=\left[0, \alpha_{0}\right] \cup\left[\alpha_{0},+\infty\right)$ with $\alpha_{0}>\alpha-1$ and let $f \in E_{\infty}$. Then condition (29) shows that $y \in E_{\infty}$. Also by the controllability assumption, we can choose $f$ such that $x\left(\alpha_{0}\right)$ is arbitrary and the resultant response on $\left[\alpha_{0},+\infty\right)$ can then be calculated. The uniform boundedness of the response and the observability assumption now establish that $A+B_{0}\left(\zeta^{-1} I_{m}-\right.$ $\left.D_{0}\right)^{-1} C$ is Hurwitz, i.e., all solutions in $\rho$ of $\operatorname{det} \mid \rho \mathrm{I}_{\mathbf{n}}-\mathrm{A}-$ $\mathrm{B}_{0}\left(\zeta \mathrm{I}_{\mathrm{m}}-\mathrm{D}_{0}\right)^{-1} \mathrm{C} \mid=0$, have modulus strictly less than unity for all choices of the complex scalar $\zeta$ in the range $\{\zeta:|\zeta| \geq \lambda\}$ Also we have that

$$
\begin{aligned}
& \operatorname{det}\left|\rho \mathrm{I}_{\mathbf{n}}-\left(\mathrm{A}+\mathrm{B}_{0}\left(\zeta \mathrm{I}_{\mathrm{m}}-\mathrm{D}_{0}\right)^{-1} \mathrm{C}\right)\right|= \\
& \frac{\operatorname{det}\left(\rho \mathrm{I}_{\mathrm{n}}-\mathrm{A}\right) \operatorname{det}\left(\zeta \mathrm{I}_{\mathrm{m}}-\mathrm{G}(\rho)\right)}{\operatorname{det}\left(\zeta \mathrm{I}_{\mathrm{m}}-\mathrm{D}_{0}\right)}
\end{aligned}
$$

which, noting the assumptions on $r\left(D_{0}\right)$ and $r(A)$, leads to condition (b) here on application of the multivariable Nyquist stability criterion in the discrete domain. This completes the proof of necessity.

To prove sufficiency, first note that condition (a) and the fact that the spectrum of $L_{\alpha}$ in this case is independent of $\alpha$ ensures that $r_{\infty}=r\left(L_{\alpha_{0}}\right)<1$ for any $\alpha_{0}>\alpha-1$. Now consider the equation $\left(\zeta I-L_{\alpha}\right) y=f$. Condition (a) ensures that the map $\left(\zeta I-L_{\alpha}\right)^{-1}: f \mapsto y$ in $\ell_{2}^{m}\left[0, \alpha_{0}\right]$ is uniformly bounded on any interval $\left[0, \alpha_{0}\right]$ with $\alpha_{0}>\alpha-1$. Condition (b) shows that the matrix $A+B_{0}\left(\zeta I_{m}-D_{0}\right)^{-1} C$ is bounded and stable for all complex $\zeta$ in the set $\{\zeta:|\zeta| \geq \lambda\}$ where $\lambda$ is any point in the nonempty set $\left(\sup _{|\rho|=1} r(G(\rho)), 1\right)$. Hence for these values of $\zeta \exists N>0$ and $\epsilon \in(0,1)$ such that $\left\|\left(A+B_{0}\left(\zeta I_{m}-D_{0}\right)^{-1} C\right)^{p}\right\| \leq N \epsilon^{p}, \forall p \geq 0, \forall|\zeta| \geq \lambda$. The map $f \mapsto y$ in $\ell_{2}^{m}\left[\alpha_{0}, \alpha\right)$ is hence uniformly bounded over the infinite range $\alpha \geq \alpha_{0}$ and $\{\zeta:|\zeta| \geq \lambda\}$. Condition (29) follows by combining these results.

As to be expected intuitively, stability along the pass demands that $r(A)<1$, i.e., the first pass profile is uniformly bounded with respect to the pass length. The simple example with limit profile given by (26) demonstrates, however, that this condition is necessary but not sufficient (only the condition on $G(\rho)$ is violated if $|\beta| \geq 1$ ). Each of these conditions for stability along the pass also has a well defined physical interpretation as summarized next.

1) Asymptotic stability demands that the sequence $\left\{y_{k}(0)\right\}_{k \geq 1}$, i.e., the sequence formed by evaluating the pass profile at $p=0$ on each pass does not become unbounded in a well defined sense as the pass number $k \rightarrow+\infty$.

2) The requirement that $r(A)<1$ demands that the first pass profile is uniformly bounded with respect to the pass length.

3) The last condition effectively demands that each frequency component of the initial pass profile is attenuated from pass to pass.

\section{1-D EQUiVALENT MODEL AND StABILITY TEST}

In this section, we use a previously developed 1-D equivalent state-space model description of the dynamics of discrete linear repetitive processes described by (1) and (3) to develop new stability tests which can be implemented by direct application of 1-D linear systems tests compatible with computer aided analysis. As necessary background, we first summarize the construction of the 1-D equivalent model.

Consider a discrete linear repetitive process described by (1) with boundary conditions of the form (3). Then the basic steps in the construction of the 1-D equivalent model of the dynamics of this process are given below - for a detailed treatment see [7].

Step 1) The basic state-space model (1) is first transformed to the standard 2-D linear systems Roesser state-space model by applying a simple 'forward transformation' of the pass profile vector followed by a change of variable in the pass number. In particular, set

$y_{k-1}(p):=v_{k}(p), k \geq 0,0 \leq p \leq \alpha-1, l:=k+1$

respectively. Then, on introducing these substitutions into (1) the dynamics of a discrete linear repetitive process can be equivalently described by the standard (also termed regular or nonsingular) Roesser state-space model

$$
\begin{aligned}
x_{l}(p+1) & =A x_{l}(p)+B u_{l}(p)+B_{0} v_{l}(p) \\
v_{l+1}(p) & =C x_{l}(p)+D u_{l}(p)+D_{0} v_{l}(p)
\end{aligned}
$$

where $l=1,2, \ldots, p=0,1,2, \ldots, \alpha-1$.

Step 2) To calculate the process response using this equivalent description it is necessary to 'transform' and apply the 
correct set of boundary conditions. In the case of (3) these become

$$
\begin{aligned}
& x_{l}(0)=d_{l}+\sum_{j=0}^{\alpha-1} K_{j} v_{l}(j), l=1,2, \cdots \\
& v_{1}(p)=y(p), p=0,1,2, \ldots, \alpha-1 .
\end{aligned}
$$

Step 3) Now define the so-called global pass profile, state and where input super-vectors for (1) as

$$
\begin{aligned}
\mathbf{Y}(l) & :=\left[\begin{array}{c}
v_{l}(0) \\
v_{l}(1) \\
\vdots \\
v_{l}(\alpha-1)
\end{array}\right] \\
\mathbf{X}(l) & :=\left[\begin{array}{c}
x_{l}(1) \\
\vdots \\
x_{l}(\alpha)
\end{array}\right] \\
\mathbf{U}(l) & :=\left[\begin{array}{c}
u_{l}(0) \\
u_{l}(1) \\
\vdots \\
u_{l}(\alpha-1)
\end{array}\right] .
\end{aligned}
$$

Also, define the matrices $\tilde{\Phi}, \Delta, \Theta$ and $\tilde{\Gamma}, \Sigma, \Psi$ as shown in (36)-(39) at the bottom of the page.
Introducing these substitutions now leads immediately to the following 1-D equivalent model for the dynamics of discrete linear repetitive processes described by (1) and (3)

$$
\begin{aligned}
\mathbf{Y}(l+1) & =\Phi \mathbf{Y}(l)+\Delta \mathbf{U}(l)+\Theta d_{l} \\
\mathbf{X}(l) & =\Gamma \mathbf{Y}(l)+\Sigma \mathbf{U}(l)+\Psi d_{l}
\end{aligned}
$$

$$
\begin{aligned}
\Phi & =[\tilde{\Phi}+\Theta K]_{m \alpha \times m \alpha} \\
\Gamma & =[\tilde{\Gamma}+\Psi K]_{n \alpha \times m \alpha} \\
K & =\left[K_{0} K_{1} \cdots K_{\alpha-1}\right]_{n \times m \alpha} .
\end{aligned}
$$

Hence we get (45) and (46) shown at the bottom of the next page.

Previous research in the 2-D/nD systems area has also developed a 1-D equivalent model for the underlying dynamics—see, for example, [3]. A key point about the resulting model is that it is 'time varying' in the sense that the dimensions of the matrices and vectors which define it increase as the process evolves over the positive quadrant, and this fact alone has prevented its effective use in the development of a mature systems theory for the $2-\mathrm{D} / \mathrm{nD}$ processes it describes. This feature is not present in the equivalent 1-D model for discrete linear repetitive processes

$$
\begin{aligned}
& \tilde{\Phi}=\left[\begin{array}{ccccc}
D_{0} & 0 & 0 & \ldots & 0 \\
C B_{0} & D_{0} & 0 & \ldots & 0 \\
C A B_{0} & C B_{0} & D_{0} & \ldots & 0 \\
\vdots & \vdots & \vdots & \ddots & \vdots \\
C A^{\alpha-2} B_{0} & C A^{\alpha-3} B_{0} & C A^{\alpha-4} B_{0} & \ldots & D_{0}
\end{array}\right]_{m \alpha \times m \alpha} \\
& \Delta=\left[\begin{array}{ccccc}
D & 0 & 0 & \ldots & 0 \\
C B & D & 0 & \ldots & 0 \\
C A B & C B & D & \ldots & 0 \\
\vdots & \vdots & \vdots & \ddots & \vdots \\
C A^{\alpha-2} B & C A^{\alpha-3} B & C A^{\alpha-4} B & \ldots & D
\end{array}\right]_{m \alpha \times r \alpha} \\
& \Theta=\left[\begin{array}{c}
C \\
C A \\
\vdots \\
C A^{\alpha-1}
\end{array}\right]_{m \alpha \times n} \\
& \tilde{\Gamma}=\left[\begin{array}{cccc}
B_{0} & 0 & \ldots & 0 \\
A B_{0} & B_{0} & \ldots & 0 \\
\vdots & \vdots & \ddots & \vdots \\
A^{\alpha-1} B_{0} & A^{\alpha-2} B_{0} & \ldots & B_{0}
\end{array}\right]_{n \alpha \times m \alpha} \\
& \Sigma=\left[\begin{array}{cccc}
B & 0 & \ldots & 0 \\
A B & B & \ldots & 0 \\
\vdots & \vdots & \ddots & \vdots \\
A^{\alpha-1} B & A^{\alpha-2} B & \ldots & B
\end{array}\right]_{n \alpha \times r \alpha} \\
& \Psi=\left[\begin{array}{c}
A \\
A^{2} \\
\vdots \\
A^{\alpha}
\end{array}\right]_{n \alpha \times n}
\end{aligned}
$$


and hence it should be of major use in the development of a 'mature' systems theory for these processes.

In what follows, we develop new results which use the 1-D model to obtain a computationally feasible test for asymptotic stability under the pass state initial vectors of the form (3). The starting point is the following result.

Lemma 1: The asymptotic stability condition of Corollary 3 is equivalent to the requirement that $\Phi$ of (42) (or (45)) satisfies $r(\Phi)<1$

Proof: Consider (22) and note that, by application of suitable block elementary operations which leave the determinant invariant, this is equivalent to

$\operatorname{det}\left(\begin{array}{ccccc}I_{n} & -W & \cdots & 0 & 0 \\ 0 & I_{n} & \ddots & \ddots & 0 \\ \vdots & \vdots & \ddots & \ddots & \vdots \\ 0 & 0 & \cdots & I_{n} & -W \\ -W_{\alpha-1} & -W_{\alpha-2} & \cdots & -W_{1} & \zeta I_{n}-W_{0}\end{array}\right) \neq 0$

where

$$
\begin{aligned}
W & =A+B_{0}\left(\zeta I_{m}-D_{0}\right)^{-1} C \\
W_{j} & =K_{j} \zeta\left(\zeta I_{m}-D_{0}\right)^{-1} C, \quad 0 \leq j \leq \alpha-1 .
\end{aligned}
$$

Now multiply the first $\alpha-1$ block rows by the scalar $\zeta$ (which does not add any nonzero roots) and then perform the following block elementary operations.

1) Multiply the last block row from the left by the matrix $A$ and add the result to the preceding block row.

2) Multiply the penultimate row of the matrix resulting from 1) from the left by $A$ and add it to the block row above.

3 ) Continue until the operation of 2) is applied to the first two block rows.

This shows that (47) is equivalent to (50) shown at the bottom of the page $\forall|\zeta| \geq 1$ where

$$
\psi=\zeta\left(\zeta I_{m}-D_{0}\right)^{-1}
$$

We can now write (50) in the form

$$
\operatorname{det}\left(\zeta I_{n \alpha}-P R\right) \neq 0, \forall|\zeta| \geq 1
$$

where $P$ is of dimension $\alpha n \times \alpha m$ and $R=\operatorname{diag}_{\alpha}\{C\}$. Hence by a standard fact from the theory of determinants $\operatorname{det}\left(\zeta I_{n \alpha}-P R\right)=\zeta^{(n-m) \alpha} \operatorname{det}\left(\zeta I_{m \alpha}-R P\right)$ and therefore (47) is equivalent to (53) shown at the bottom of the page $\forall|\zeta| \geq 1$. Finally, extract the matrix $\psi$ on the right-hand side from each block row (and note that $r\left(D_{0}\right)<1$ ) and the proof is completed by applying appropriate block row and column permutations.

$$
\begin{aligned}
& \Phi=\left[\begin{array}{cccc}
D_{0}+C K_{0} & C K_{1} & \ldots & C K_{\alpha-1} \\
C\left(B_{0}+A K_{0}\right) & \left(D_{0}+C A K_{1}\right) & \cdots & C A K_{\alpha-1} \\
C A\left(B_{0}+A K_{0}\right) & C\left(B_{0}+A^{2} K_{1}\right) & \ldots & C A^{2} K_{\alpha-1} \\
\vdots & \vdots & \ddots & \vdots \\
C A^{\alpha-2}\left(B_{0}+A K_{0}\right) & C A^{\alpha-3}\left(B_{0}+A^{2} K_{1}\right) & \ldots & \left(D_{0}+C A^{\alpha-1} K_{\alpha-1}\right)
\end{array}\right] \\
& \Gamma=\left[\begin{array}{cccc}
B_{0}+A K_{0} & A K_{1} & \ldots & A K_{\alpha-1} \\
A\left(B_{0}+A K_{0}\right) & \left(B_{0}+A^{2} K_{1}\right) & \cdots & A^{2} K_{\alpha-1} \\
A^{2}\left(B_{0}+A K_{0}\right) & A\left(B_{0}+A^{2} K_{1}\right) & \ldots & A^{3} K_{\alpha-1} \\
\vdots & \vdots & \ddots & \vdots \\
A^{\alpha-1}\left(B_{0}+A K_{0}\right) & A^{\alpha-2}\left(B_{0}+A^{2} K_{1}\right) & \ldots & \left(B_{0}+A^{\alpha} K_{\alpha-1}\right)
\end{array}\right]
\end{aligned}
$$

$\operatorname{det}\left(\begin{array}{cccc}\zeta I_{n}-A^{\alpha-1} K_{\alpha-1} \psi C & -A^{\alpha-1} K_{\alpha-2} \psi C-B_{0} \psi C & \cdots & -A^{\alpha-1} K_{0} \psi C-A^{\alpha-2} B_{0} \psi C \\ \vdots & \vdots & \vdots & \vdots \\ -A K_{\alpha-1} \psi C & -A K_{\alpha-2} \psi C & \cdots & -A K_{0} \psi C-B_{0} \psi C \\ -K_{\alpha-1} \psi C & -K_{\alpha-2} \psi C & \cdots & \zeta I_{n}-K_{0} \psi C\end{array}\right) \neq 0$

$\operatorname{det}\left(\begin{array}{cccc}\zeta I_{m}-C A^{\alpha-1} K_{\alpha-1} \psi & -C B_{0} \psi-C A^{\alpha-1} K_{\alpha-2} \psi & \cdots & -C A^{\alpha-2} B_{0} \psi-C A^{\alpha-1} K_{0} \psi \\ \vdots & \vdots & \vdots & \vdots \\ -C A K_{\alpha-1} \psi & -C A K_{\alpha-2} \psi & \cdots & C B_{0}-C A K_{0} \psi \\ -C K_{\alpha-1} \psi & -C K_{\alpha-2} \psi & \cdots & \zeta I_{m}-C K_{0} \psi\end{array}\right) \neq 0$ 
The following result now gives a set of conditions for stability along the pass of processes described by (1) and (3) which can be tested by direct application of 1-D linear systems stability tests which are compatible with a computer aided analysis environment.

Theorem 3: The linear repetitive process $S$ generated by (1) and (3) with $r\left(D_{0}\right)<1$ is stable along the pass if, and only if

1) all eigenvalues of the transfer function matrix

$$
\hat{G}(\zeta)=K\left(\zeta I_{m \alpha}-\tilde{\Phi}\right)^{-1} \Theta
$$

have modulus strictly less than unity $\forall|\zeta|=1$;

2) $r(A)<1$

3) all eigenvalues of the transfer function matrix $G(\rho)$ defined by (30) have modulus strictly less than unity $\forall|\rho|=1$.

Proof: It is only required to show that condition (a) here is equivalent to the condition of Lemma 1 . To establish this, simply note that Lemma 1 requires that

$$
\begin{aligned}
& \operatorname{det}\left(\zeta \mathrm{I}_{\mathrm{m} \alpha}-\Phi\right)=\operatorname{det}\left(\zeta \mathrm{I}_{\mathrm{m} \alpha}-\tilde{\Phi}-\Theta \mathrm{K}\right)=\operatorname{det}\left(\zeta \mathrm{I}_{\mathrm{m} \alpha}-\tilde{\Phi}\right) \\
& \quad \times \operatorname{det}\left(I_{m \alpha}-\left(\zeta I_{m \alpha}-\tilde{\Phi}\right)^{-1} \Theta K\right) \neq 0 \quad \forall|\zeta| \geq 1
\end{aligned}
$$

where the inverse used exists since $r\left(D_{0}\right)<1$ (note the structure of $\tilde{\Phi}$ of (36)). Equivalently, we require that

$$
\operatorname{det}\left(I_{m \alpha}-\left(\zeta I_{m \alpha}-\tilde{\Phi}\right)^{-1} \Theta K\right) \neq 0 \quad \forall|\zeta| \geq 1
$$

and the result now follows immediately on application of a standard relationship from the theory of determinants.

\section{Controllability}

In this section, we use the 1-D model to develop new results which completely characterize so-called complete pass controllability for discrete linear repetitive processes with boundary conditions of the form (3). As for other classes of 2-D linear systems, the situation re controllability for discrete linear repetitive processes, with any form of boundary conditions, is somewhat more complex than in the 1-D case. In particular, at a general level, so-called point and pass controllability can be formulated. In [6] the former property, essentially the existence of an admissible control sequence which will drive the system to prescribed dynamics at a particular point on a particular pass, was completely characterized in terms of rank properties of matrices with constant entries. This used a 2-D Fornasini Marchesini state-space model equivalent description of the underlying dynamics. It was also shown there that it was not possible to use this approach to characterize pass controllability. Here we show that this task is possible using the 1-D equivalent model where the resulting conditions are in easily checkable form.

The analysis which follows requires the following ordering of two tuple integers:

$$
\begin{aligned}
& (f, h) \leq(k, p) \text { iff } f \leq k \text { and } h \leq p \\
& (f, h)=(k, p) \text { iff } f=k \text { and } h=p \\
& (f, h)<(k, p) \text { iff }(f, h) \leq(k, p) \text { and }(f, h) \neq(k, p) .
\end{aligned}
$$

Also for $(a, b)<(c, d)$, the rectangle $[(a, b),(c, d)]$ is defined as follows:

$$
[(a, b),(c, d)]:=\{(a, b) \leq(i, j) \leq(c, d)\} .
$$

Now, we proceed to the pass controllability property and (in the following sub-section) so-called pass state controllability.

\section{A. Pass Controllability}

The property of so-called pass controllability for discrete linear repetitive processes is defined as follows.

Definition 1: The discrete linear repetitive processes described by (1) and (3) are said to be simultaneously, or pass, controllable if $\exists$ a pass number $K^{*}$ and control input vectors defined on the rectangle $\left[(0,0),\left(K^{*}, \alpha-1\right)\right]$ which will drive the process to an arbitrarily specified pass profile on pass $K^{*}$.

It is routine to see that pass controllability of the dynamics of processes described by (1) and (3) is equivalent to controllability of the equivalent 1-D model, i.e., the pair $\{\Phi, \Delta\}$ is controllable. This is stated formally as the following result.

Theorem: Discrete linear repetitive processes described by (1) and (3) are pass controllable if, and only if

$$
\operatorname{rank}[z I-\Phi, \Delta]=m \alpha \quad \forall \zeta \in \mathcal{C} .
$$

The condition of (59) can be decomposed into a simpler structure equivalent form by introducing the following matrices:

$$
\begin{aligned}
\Omega_{0}= & {\left[C K_{1}, C K_{2}, \cdots, C K_{\alpha-1}, D\right] } \\
\Omega_{1}= & {\left[C\left(B_{0}+A K_{0}\right), C A K_{2}, \cdots, C A K_{\alpha-1}, C B, D\right] } \\
& \vdots \\
\Omega_{\alpha-1}= & {\left[C A^{\alpha-2}\left(B_{0}+A K_{0}\right), \cdots, C\right.} \\
& \times\left(B_{0}+A^{\alpha-2} K_{\alpha-2}\right), C A^{\alpha-2} B, C A^{\alpha-3} B, C A^{\alpha-4} B, \\
& \cdots C A B, C B, D] .
\end{aligned}
$$

Then, we have the following result.

Corollary 4: Discrete linear repetitive processes described by (1) and (3) are pass controllable if, and only if, $\forall j=0,1, \cdots, \alpha-1$

$$
\operatorname{rank}\left[\zeta I_{m}-\left(D_{0}+C A^{j} K_{j}\right), \Omega_{j}\right]=m \quad \forall \zeta \in \mathcal{C} \text {. }
$$

Proof: The set of conditions given in (61) are, in fact, equivalent to the necessary and sufficient condition of (59) since they are obtained from it by an obvious decomposition of this condition.

The following corollary holds for the case when the pass state initial vector sequence has the simpler form of (2).

Corollary 5: Discrete linear repetitive processes described by (1) and (2) are pass controllable if, and only if, the pair $\left\{D_{0}, D\right\}$ is controllable.

This result shows that the property of pass controllability cannot be present if $D_{0}=0$ or $D=0$.

In the case of dynamic boundary conditions of the form (3), the matrix $\Phi$ has a somewhat more complex structure than $\tilde{\Phi}$. In particular, it is the lower block triangular structure of $\tilde{\Phi}$ which enables the simple necessary and sufficient conditions of Corollary 5 to be derived for pass controllability under boundary conditions of the form (2). It is, however, also possible to derive 'relatively simple structure' conditions for pass controllability in the presence of boundary conditions of the form of (3). 
Theorem 5: Discrete linear repetitive processes described by (1) and (3) are pass controllable if the following conditions hold $\forall \zeta \in \mathcal{C}$

$$
\begin{array}{r}
\operatorname{rank}\left[\zeta I_{m}-\left(D_{0}+C K_{0}\right), D\right]=m, \\
\operatorname{rank}\left[\zeta I_{m}-\left(D_{0}+C A K_{1}\right), D\right]=m, \\
\vdots \\
\operatorname{rank}\left[\zeta I_{m}-\left(D_{0}+C A^{\alpha-1} K_{\alpha-1}\right), D\right]=m .
\end{array}
$$

Proof: This is an immediate consequence of the fact that the matrix $\Delta$ has, see (37), a lower block triangular structure in this case.

From the above analysis, we see the essential difference between pass controllability of processes with boundary conditions of the form (2) and (3) respectively. In the former case, it is easy to see that the pass controllability horizon, i.e., the number of passes $K^{*}$ required to attain the prescribed pass profile, can be equal to $m-$ the dimension of the pass profile vector. This property is due to the block triangular structure of the matrices (36) and (37) which define this property for processes described by (1) and (2). In the latter case, however, the pass controllability horizon must generally be assumed to be equal to the full (state) dimension of the 1-D equivalent model, i.e., $m \alpha$.

As a simple example to highlight the critical role of the boundary conditions on the pass controllability properties of linear repetitive processes of the form (1), consider the case when $\alpha=2$ and

$$
C=\left[\begin{array}{ll}
1 & 1
\end{array}\right], \quad D=\left[\begin{array}{l}
1 \\
2
\end{array}\right], \quad D_{0}=\left[\begin{array}{ll}
0 & 1 \\
1 & 0
\end{array}\right] .
$$

This example is pass controllable when the boundary conditions are of the simple form (2), but if they are of the form (3), i.e., $K=\left[K_{0}, K_{1}\right]$ then it is easy to see that pass controllability does not hold when $K_{0}+2 K_{1}=-3$. Equally, it is possible that a process with boundary conditions of the form (2) is not pass controllable but can be made so by suitable choice of the boundary conditions of the form (3).

In common with the 1-D case, transformations of both the state and/or pass profile vector play an important role in the analysis of discrete linear repetitive processes. The most basic form of these are defined next where $T$ and $S$ are nonsingular matrices of dimensions $n \times n$ and $m \times m$, respectively

$$
\begin{aligned}
& x_{k+1}(t)=T \hat{x}_{k+1}(t) \\
& y_{k+1}(t)=S \hat{y}_{k+1}(t) .
\end{aligned}
$$

These are termed similarity transformations.

Theorem 6: Pass controllability of processes described by (1) and (3) is invariant under the similarity transformations defined by (64)

Proof: Applying (64) to (1) and (3) gives

$$
\begin{aligned}
\hat{x}_{k+1}(p+1)= & T^{-1} A T \hat{x}_{k+1}(p) \\
& +T^{-1} B u_{k+1}(t)+T^{-1} B_{0} S \hat{y}_{k}(p) \\
\hat{y}_{k+1}(p)= & S^{-1} C T \hat{x}_{k+1}(p) \\
& +S^{-1} D u_{k+1}(p)+S^{-1} D_{0} S \hat{y}_{k}(p) \\
\hat{x}_{k+1}(0)= & T^{-1} d_{k+1}+\sum_{j=0}^{\alpha-1} T^{-1} K_{j} S \hat{y}_{k}(j) .
\end{aligned}
$$

Hence, in the 1-D model the matrices $\Phi$ and $\Delta$ are transformed to

$$
\Phi^{*}=\hat{S}^{-1} \Phi \hat{S}, \text { and } \Delta^{*}=\hat{S}^{-1} \Delta
$$

respectively, where $\hat{S}=\operatorname{diag}\{S, S, \cdots, S\}$, and this completes the proof.

\section{B. Pass State Controllability}

The property of pass state controllability is defined as follows.

Definition 2: The discrete linear repetitive processes described by (1) and (3) are said to be simultaneously, or pass, state controllable if $\exists$ a pass number $K^{*}$ and control input vectors defined on the rectangle $\left[(0,0),\left(K^{*}, \alpha-1\right)\right]$ which will drive the process to an arbitrarily specified state vector sequence on pass $K^{*}$.

In order to study this property, consider first the equivalent 2-D Roesser state-space model representation of the dynamics of discrete linear repetitive processes given by (33). Then in this 2-D model, the current pass state vector of the repetitive process is treated as a local horizontal state sub-vector and the pass profile vector as a local vertical state sub-vector. Previously, after introducing the 1-D state-space model we have investigated only pass controllability relative to the global pass profile vector as it represents the global state of the process. The subject of this sub-section is the pass controllability properties of global state vector $\mathbf{X}(l)$ of (35). Note that it is never possible to simultaneously achieve arbitrarily specified pass profile and state vector sequences along a given pass, i.e., the processes considered in this paper can never be simultaneously pass and pass state controllable.

It is clear from (40) and (41) that $\mathbf{X}(l)$ depends statically on the global pass profile vector $\mathbf{Y}(l)$, since its dynamics have been 'absorbed' into the matrices which define the 1-D equivalent model. Hence, $\mathbf{X}(l)$ alone cannot be the subject of the controllability investigations. However, in applications we may only be interested in reaching just the specified trajectory $\mathbf{X}(l)$ and not $\mathbf{Y}(l)$. The conditions under which this can be achieved are established next.

The only way of obtaining a pre-defined set of state vectors along a pre-specified pass number, say $N$, is to first obtain the prescribed pass profile on pass number $N$, say $Y^{*}$,

$$
\begin{aligned}
Y^{*} & =Y(l+1)_{l=N}=Y(N+1) \\
& =\tilde{\Phi} Y(N)+\Delta U(N)+\Theta X(N, 0)
\end{aligned}
$$

such that $\exists$ on the next pass $N+1$ a control input vector $U(N+$ 1) which gives the required $X^{*}$, i.e.,

$X^{*}=X(N+1)=\tilde{\Gamma} Y(N+1)+\Sigma U(N+1)+\Psi X(N+1,0)$.

The initial state vector $X(N+1,0)$ is pre-specified on each pass, i.e., it not the subject of any control action. Consequently introduce

$$
\tilde{X}=X^{*}-\Psi X(N+1,0)
$$

to yield from (68)

$$
\tilde{X}=\tilde{\Gamma} Y(N+1)+\Sigma U(N+1) .
$$


The problem now is to find an admissible pass profile trajectory $Y(N+1)$ and input $U(N+1)$ such that (68) holds for a prescribed $\tilde{X}$.

The necessary and sufficient condition for a solution to this problem clearly is

$$
\operatorname{rank}[\tilde{\Gamma}, \Sigma]=\operatorname{rank}[\tilde{\Gamma}, \Sigma, \tilde{X}] .
$$

Since it is not possible to use $U(N+1)$, set this vector equal to zero and then (70) and (71) become

$$
\tilde{X}=\tilde{\Gamma} Y(N+1)
$$

and

$$
\operatorname{rank}[\tilde{\Gamma}]=\operatorname{rank}[\tilde{\Gamma}, \tilde{X}]
$$

respectively. From this is follows that to obtain a given $Y^{*}$ we must have pass controllability, i.e., $\left\{D_{0}, D\right\}$ must be a controllable pair.

Given that we can only obtain either $X^{*}$ or $Y^{*}$, it follows in the former case that the pass profile on the next pass will be given by

$Y^{* *}=Y(N+2)=\tilde{\Phi} Y(N+1)+\Delta U(N+1)+\Theta X(N+1,0)$.

Also, the block structure of the matrices in the above equation can be exploited to good effect. In particular, write

$$
\begin{aligned}
\tilde{X} & =\left[\begin{array}{c}
\tilde{X}_{1} \\
\vdots \\
\tilde{X}_{\alpha}
\end{array}\right]=X^{*}-\Psi X(N+1,0) \\
& =\left[\begin{array}{c}
X(N+1,1)-A X(N+1,0) \\
\vdots \\
X(N+1, \alpha)-A^{\alpha} X(N+1,0)
\end{array}\right] .
\end{aligned}
$$

Then, we can write the first sub-equation in (70) as

$$
B_{0} Y(N+1,0)+B U(N+1,0)=\tilde{X}_{1}
$$

and the solvability condition for this equation is

$$
\operatorname{rank}\left[B_{0}, B\right]=\operatorname{rank}\left[B_{0}, B, \tilde{X}_{1}\right] .
$$

If this condition is satisfied let $Y^{*}(N+1,0)$ and $U^{*}(N+1,0)$ be the corresponding solution.

The second sub-equation in (70) can now be written as

$$
\begin{aligned}
A B_{0} Y^{*}(N & +1,0)+B_{0} Y(N+1,1) \\
& +A B U^{*}(N+1,0)+B U(N+1,1)=\tilde{X}_{2}
\end{aligned}
$$

or

$$
B_{0} Y(N+1,1)+B U(N+1,1)=\hat{X}_{2}
$$

where

$$
\hat{X}_{2}=\tilde{X}_{2}-A B_{0} Y^{*}(N+1,0)-A B U^{*}(N+1,0) .
$$

Also, (79) has a solution if, and only if,

$$
\operatorname{rank}\left[B_{0} B\right]=\operatorname{rank}\left[B_{0} B \hat{X}_{2}\right]
$$

and let $Y^{*}(N+1,1)$ and $U^{*}(N+1,1)$ be its solution under this condition. Now continue this solution process until point $\alpha-1$, when we have

$$
B_{0} Y(N+1, \alpha-1)+B U(N+1, \alpha-1)=\hat{X}_{\alpha}
$$

where

$$
\begin{aligned}
& \hat{X}_{\alpha}=\tilde{X}_{\alpha}-\sum_{i=1}^{\alpha-1}\left(A^{i} B_{0} Y^{*}(N+1, \alpha-1)\right. \\
&\left.-A^{i} B U^{*}(N+1, \alpha-i-1)\right) .
\end{aligned}
$$

Hence, if the matrix $\left[B_{0} B\right]$ is of full row rank $(n)$ then (70) always has a solution and an arbitrary pass state vector can be reached. If this rank condition does not hold, only an appropriate combination of pass states can be reached.

Suppose now that we wish to reach $X^{*}$ without control action on the last pass. Then, in this case, the first sub-equation of (70) can be written as

$$
B_{0} Y(N+1,0)=\tilde{X}_{1}
$$

and this condition has a solution when

$$
\operatorname{rank}\left[B_{0}\right]=\operatorname{rank}\left[B_{0}, \tilde{X}_{1}\right] .
$$

If this condition holds, let $Y^{*}(N+1,0)$ be its solution. Also the second sub-equation can be written as

$$
B_{0} Y(N+1,1)=\hat{X}_{2}
$$

where

$$
\hat{X}_{2}=\tilde{X}_{2}-A B_{0} Y^{*}(N+1,0)
$$

which has a solution if, and only if,

$$
\operatorname{rank}\left[B_{0}\right]=\operatorname{rank}\left[B_{0}, \hat{X}_{2}\right] .
$$

Suppose also that (88) holds and let $Y^{*}(N+1,1)$ be its solution. Then continuing this process until point $\alpha-1$ gives

$$
B_{0} Y(N+1, \alpha-1)=\hat{X}_{\alpha}
$$

where

$$
\hat{X}_{\alpha}=\tilde{X}_{\alpha}-\sum_{i=1}^{\alpha-1} A^{i} B_{0} Y^{*}(N+1, \alpha-1-i) .
$$

From this, it follows that if $B_{0}$ has full row rank ( $n$ ) then (70) always has a solution without control action on the last pass and any pass state vector can be reached. If this condition does not 
hold then only an appropriate linear combination of such states may be reached.

\section{Stabilization by Choice of the Pass State Initial VECTOR SEQUENCE}

A major question which now arises is: Under what conditions can we select a set of matrices $K_{j}, 0 \leq j \leq \alpha-1$, such that the process (1) is asymptotically stable under the boundary conditions (3) defined by this choice?

This question is of particular relevance in the optimal control application (see [8]). As shown below, this problem can be solved using the theory of 1-D discrete linear systems pole placement by state feedback.

In essence, the problem here is one of finding a matrix $K$ such that the eigenvalues of $\tilde{\Phi}+\Theta K$ can be assigned to arbitrary locations inside the unit circle in the complex plane, i.e., the pole placement problem for a 1-D discrete linear system with state matrix $\tilde{\Phi}$, input matrix $\Theta$, and state feedback matrix $K$. Now introduce the following new controllability concept which is termed initial state pass controllability.

Definition 3: Discrete linear repetitive processes described by (1) and (3) are said to be initial state pass controllable provided

$$
\operatorname{rank}\left[\zeta I_{m \alpha}-\tilde{\Phi}, \Theta\right]=m \alpha \quad \forall \zeta \in \mathbb{C}
$$

Now, we can establish the following theorem.

Theorem 7: Consider discrete linear repetitive processes described by (1) and (3). Then $\exists$ an appropriate choice of matrices $K_{j}, 0 \leq j \leq \alpha-1$, and hence the matrix $K$ of (44), such that the eigenvalues of the matrix $\Phi=\tilde{\Phi}+\Theta K$ defined by (42) can be assigned to arbitrary locations in the complex plane if, and only if, the example under consideration is initial state pass controllable.
Proof: Follows immediately from the theory of the 1-D discrete linear invariant pole placement problem with state matrix $\tilde{\Phi}$, input matrix $\Theta$ and state feedback matrix $K$.

At this stage, it is instructive to consider the single-input single-output case when (91) takes the form of (92) shown at the bottom of the page. It now follows immediately that this condition holds when $\zeta \neq D_{0}$. In the case when $D_{0}=0$, (92) reduces to (93) shown at the bottom of the page, where $C \neq 0$. Suppose also $\exists$ an integer $0 \leq t \leq n-1$ such that

$$
C A^{t} B_{0} \neq 0 \text {. }
$$

Also let $\tilde{t}$ be the minimum value of $t$ for which (94) holds and hence $C A^{t} B_{0}=0, t<\tilde{t}$. Then it is easy to see that (94) in this case requires that

$$
\operatorname{rank}\left[\begin{array}{c}
C \\
C A \\
\vdots \\
C A^{\tilde{t}-1}
\end{array}\right]=\tilde{t} .
$$

In 1-D linear systems terms, this last result is equivalent to the requirement that the minimum dimension of the observability subspace for a discrete 1-D linear system with state matrix $A$ and output matrix $C$ is equal to $\tilde{t}$. Also, by the Cayley-Hamilton theorem, it follows that if there is no $0 \leq \tilde{t} \leq n-1$, then $C A^{t} B_{0}=0, \forall t$. In what follows, we extend this analysis to the multiple-input multiple-output case by use of similarity transforms which produce the Jordan canonical form for the matrix $D_{0}$.

For ease of notation, let $Q$ denote the matrix on the left-hand side of (91). Then we require to check that $\operatorname{rank}(Q)=m \alpha$ for $z=\gamma_{i}, 1 \leq i \leq m$, where $\gamma_{i}$ is an eigenvalue of $D_{0}$. Here, as an alternative to numerical checking, we proceed by using the Schur decomposition of $D_{0}$. In particular, we use orthogonal or unitary matrices to implement the Schur decomposition, i.e., the

$$
\begin{aligned}
\operatorname{rank}\left[\begin{array}{cccccc}
\zeta-D_{0} & 0 & 0 & \cdots & 0 & C \\
-C B_{0} & \zeta-D_{0} & 0 & \cdots & 0 & C A \\
C A B_{0} & -C B_{0} & \zeta-D_{0} & \cdots & 0 & C A^{2} \\
\vdots & \vdots & \vdots & \ddots & \cdots & \vdots \\
-C A^{\alpha-2} B_{0} & -C A^{\alpha-3} B_{0} & -C A^{\alpha-4} B_{0} & \cdots & \zeta-D_{0} & C A^{\alpha-1}
\end{array}\right] \\
=\alpha, \forall \zeta \in \mathbb{C}
\end{aligned}
$$

$$
\operatorname{rank}\left[\begin{array}{cccccc}
C & 0 & 0 & \cdots & 0 & 0 \\
C A & -C B_{0} & 0 & \cdots & 0 & 0 \\
C A^{2} & -C A B_{0} & -C B_{0} & \cdots & 0 & 0 \\
\vdots & \vdots & \vdots & \ddots & \vdots & \vdots \\
C A^{\alpha-1} & -C A^{\alpha-2} B_{0} & -C A^{\alpha-3} B_{0} & \cdots & -C B_{0} & 0
\end{array}\right]=\alpha
$$


matrix $S$ transforming the pass profile vector, see (64), satisfies $S^{-1}=S^{T}$ in the case when $D_{0}$ has only real eigenvalues, and $S^{-1}=\left(S^{*}\right)$ when this matrix has complex eigenvalues, where $*$ denotes the complex conjugate transpose operation.

Executing the Schur decomposition now transforms (91) to the requirement (96) shown at the bottom of the page, where

$$
\hat{D}_{0}=S^{*} D_{0} S, \hat{C}=S^{*} C, \hat{B}_{0}=B_{0} S .
$$

Here the matrix $\hat{D}_{0}$ is lower triangular and denote its eigenvalues by $\gamma_{i}, 1 \leq i \leq m$. Also the matrix $Q$ can now be written in block form as

$$
Q=\left[Q_{i, j}\right], i=1,2, \cdots, \alpha, j=1,2, \cdots, \alpha+1
$$

where

$$
\begin{aligned}
Q_{i, i} & =\left[\begin{array}{ccc}
z-\lambda_{1} & & 0 \\
& z-\lambda_{2} & \\
& \ddots & \\
\star & & z-\lambda_{m}
\end{array}\right] \\
Q_{i, j} & =0, j>i \text { and } j \neq \alpha+1 \\
Q_{i, \alpha+1} & =\left[\begin{array}{c}
\hat{c}_{1} A^{i-1} \\
\hat{c}_{2} A^{i-1} \\
\vdots \\
\hat{c}_{m} A^{i-1}
\end{array}\right] \\
Q_{i, j}= & -\left[\begin{array}{c}
\hat{c}_{1} A^{i-j-1} \hat{B}_{0} \\
\hat{c}_{2} A^{i-j-1} \hat{B}_{0} \\
\vdots \\
\hat{c}_{m} A^{i-j-1} \hat{B}_{0}
\end{array}\right], i>j
\end{aligned}
$$

where $\hat{c}_{i}, 1 \leq i \leq m$, denotes a row of $\hat{C}$, and in (99) $\star$ denotes the fact that this matrix is lower triangular.

Due to the quasitriangular form of $Q$ here, it is possible to obtain simpler conditions where the matrices involved have considerably reduced dimensions. In particular, introduce the $m-$ $1 \times m-1$ matrix $\Lambda_{i}$ (where $\star$ again denotes that this matrix is lower triangular) given as (103) at the bottom of the page, and also

$$
\hat{C}^{i}=\left[\begin{array}{c}
\hat{c}_{1} \\
\vdots \\
\hat{c}_{i-1} \\
\hat{c}_{i+1} \\
\vdots \\
\hat{c}_{m}
\end{array}\right], \hat{B}_{0}^{i}=\left[\begin{array}{llllll}
\hat{b}_{0}^{1} & \cdots & \hat{b}_{0}^{i-1} \hat{b}_{0}^{i+1} & \ldots & \hat{b}_{0}^{m}
\end{array}\right]
$$

which are of dimensions $m-1 \times n$ and $n \times m-1$ respectively, and $\hat{b}_{0}^{i}, 1 \leq i \leq m$, denotes a column of $\hat{B}_{0}$. Now consider the matrices $G_{i}:=\left.Q\right|_{z=\lambda_{i}}, i=1,2, \cdots, m$ which can be transformed by appropriate row and column permutations to the form

$$
\Gamma_{i}=\left[\begin{array}{lll}
\overline{C^{i} B_{0}^{i}} & \overline{C^{i} b_{0}^{i}} & \overline{C^{i} A} \\
\overline{c_{i} B_{0}^{i}} & \overline{c_{i} b_{0}^{i}} & \overline{c_{i} A}
\end{array}\right]
$$

where the matrices $\overline{C^{i} B_{0}^{i}}((m-1) \alpha \times(\alpha-1)), \overline{C^{i} b_{0}^{i}}((m-$ 1) $\alpha \times \alpha), \overline{C^{i} A}((m-1) \alpha \times n), \overline{c_{i} B_{0}^{i}}(\alpha \times(m-1) \alpha), \overline{c_{i} b_{0}^{i}}$ $(\alpha \times \alpha)$, and $\overline{c_{i} A}(\alpha \times n)$ are defined as shown in (106)-(110) at the bottom of the next page, where $V_{i, m-1}\left(V^{m-1,1}\right)$ denotes the $1 \times m-1(m-1 \times 1)$ row (column) vector whose first $l-1$ entries are nonzero and the rest are zero.

$$
\begin{aligned}
\operatorname{rank}(Q) & =\operatorname{rank}\left[\begin{array}{cccccc}
\zeta I_{m}-\hat{D}_{0} & 0 & 0 & \cdots & 0 & \hat{C} \\
-\hat{C} \hat{B}_{0} & \zeta I_{m}-\hat{D}_{0} & 0 & \cdots & 0 & \hat{C} A \\
-\hat{C} A \hat{B}_{0} & -\hat{C} \hat{B}_{0} & \zeta I_{m}-\hat{D}_{0} & \cdots & 0 & \hat{C} A^{2} \\
\vdots & \vdots & \vdots & \ddots & \vdots & \vdots \\
-\hat{C} A^{\alpha-2} \hat{B}_{0} & -\hat{C} A^{\alpha-3} \hat{B}_{0} & -\hat{C} A^{\alpha-4} \hat{B}_{0} & \cdots & \zeta I_{m}-\hat{D}_{0} & \hat{C} A^{\alpha-1}
\end{array}\right] \\
& =m \alpha, \forall \zeta \in \mathbb{C}
\end{aligned}
$$

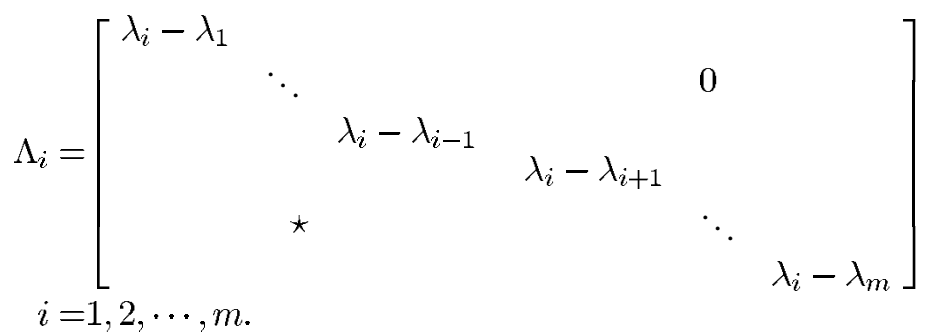


Clearly $\Lambda_{i}, i=1,2, \cdots, m$ is nonsingular and hence $\overline{C^{i} B_{0}^{i}}$ is also nonsingular. Now consider $\Gamma_{i}$, multiply the first block row on the left by $\overline{c^{i} B_{0}^{i}}\left(C^{i} B_{0}^{i}\right)^{-1}$, and subtract the result from the second block row to yield

$$
\begin{aligned}
\tilde{\Gamma}_{i}=\left[\frac{\overline{C^{i} B_{0}^{i}}}{0}\left|\frac{\overline{C^{i} b_{0}^{i}}}{\overline{c^{i} b_{0}^{i}}-\overline{c^{i} B_{0}^{i}\left(C^{i} B_{0}^{i}\right)^{-1} C^{i} b_{0}^{i}}}\right|\right. \\
\left.\overline{\overline{c^{i} A}-\overline{c^{i} B_{0}^{i}\left(C^{i} B_{0}^{i}\right)^{-1}} \overline{C^{i} A}}\right] .
\end{aligned}
$$

Then, the following result follows immediately.
Theorem 8: Discrete linear repetitive processes described by (1) and (3) are initial state pass controllable if, and only if, each of the matrices

$$
\begin{aligned}
C_{p c}: & =\left[\overline{c^{i} b_{0}^{i}}-\overline{c^{i} B_{0}^{i}}\left(\overline{C^{i} B_{0}^{i}}\right)^{-1} \overline{C^{i} b_{0}^{i}}\right. \\
& \left.\mid \overline{c^{i} A}-\overline{c^{i} B_{0}^{i}}\left(\overline{C^{i} B_{0}^{i}}\right)^{-1} \overline{C^{i} A}\right], \\
i & =1,2, \cdots, m
\end{aligned}
$$

have full row rank.

An alternative method of testing for initial state pass controllability is to decompose the requirement of (91) into 'slices,' i.e., the condition is equivalent to the set of conditions shown in (113) at the bottom of the page holding $\forall z \in \mathbb{C}$. Finally, note that any equivalent set of conditions could be substituted for each of the conditions in (113).

$$
\begin{aligned}
& \overline{C^{i} B_{0}^{i}}=\left[\begin{array}{ccccc}
\Lambda_{i} & & & & 0 \\
-\hat{C}^{i} \hat{B}_{0}^{i} & \Lambda_{i} & & & \\
-\hat{C}^{i} A \hat{B}_{0}^{i} & -\hat{C}^{i} \hat{B}_{0}^{i} & \Lambda_{i} & & \\
\vdots & \vdots & \vdots & \ddots & \\
-\hat{C}^{i} A^{\alpha-2} \hat{B}_{0}^{i} & -\hat{C}^{i} A^{\alpha-3} \hat{B}_{0}^{i} & -\hat{C}^{i} A^{\alpha-4} \hat{B}_{0}^{i} & \cdots & \Lambda_{i}
\end{array}\right] \\
& \overline{c_{i} B_{0}^{i}}=\left[\begin{array}{ccccc}
V_{1, m-1} & & & & 0 \\
-\hat{c}^{i} \hat{B}_{0}^{i} & V_{1, m-1} & & & \\
-\hat{c}^{i} A \hat{B}_{0}^{i} & -\hat{c}^{i} \hat{B}_{0}^{i} & V_{1, m-1} & & \\
\vdots & \vdots & \vdots & \ddots & \\
-\hat{c}^{i} A^{\alpha-2} \hat{B}_{0}^{i} & -\hat{c}^{i} A^{\alpha-3} \hat{B}_{0}^{i} & -\hat{c}^{i} A^{\alpha-4} \hat{B}_{0}^{i} & \cdots & V_{1, m-1}
\end{array}\right] \\
& \overline{\hat{C}^{i} b_{0}^{i}}=\left[\begin{array}{ccccc}
V_{m-1,1} & & & & 0 \\
-\hat{C}^{i} \hat{b}_{0}^{i} & V^{m-1,1} & & & \\
-\hat{C}^{i} A \hat{b}_{0}^{i} & -\hat{C}^{i} \hat{b}_{0}^{i} & V^{m-1,1} & & \\
\vdots & \vdots & \vdots & \ddots & \\
-\hat{C}^{i} A^{\alpha-2} \hat{b}_{0}^{i} & -\hat{C}^{i} A^{\alpha-3} \hat{b}_{0}^{i} & -\hat{C}^{i} A^{\alpha-4} \hat{b}_{0}^{i} & \cdots & V^{m-1,1}
\end{array}\right] \\
& \overline{c_{i} b_{0}^{i}}=\left[\begin{array}{ccccc}
0 & & & & 0 \\
-\hat{c}^{i} \hat{b}_{0}^{i} & 0 & & & \\
-\hat{c}^{i} A \hat{b}_{0}^{i} & -\hat{c}^{i} \hat{b}_{0}^{i} & 0 & & \\
\vdots & \vdots & \vdots & \ddots & \\
-\hat{c}^{i} A^{\alpha-2} \hat{b}_{0}^{i} & -\hat{c}^{i} A^{\alpha-3} \hat{b}_{0}^{i} & -\hat{c}^{i} A^{\alpha-4} \hat{b}_{0}^{i} & \cdots & 0
\end{array}\right] \\
& \overline{C^{i} A}=\left[\begin{array}{c}
\hat{C}^{i} \\
\hat{C}^{i} A \\
\hat{C}^{i} A^{2} \\
\vdots \\
\hat{C}^{i} A^{\alpha-1}
\end{array}\right], \overline{c_{i} A}=\left[\begin{array}{c}
\hat{c}^{i} \\
\hat{c}^{i} A \\
\hat{c}^{i} A^{2} \\
\vdots \\
\hat{c}^{i} A^{\alpha-1}
\end{array}\right]
\end{aligned}
$$

$$
\begin{array}{r}
\operatorname{rank}\left[z I_{m}-D_{0}, \quad C\right]=m \\
\operatorname{rank}\left[z I_{m}-D_{0}, \quad C\left[\begin{array}{ll}
B_{0} & A B_{0}
\end{array}\right]=m\right.
\end{array}
$$$$
\operatorname{rank}\left[z I_{m}-D_{0}, \quad C\left[\begin{array}{llll}
B_{0} & A B_{0} & \cdots & A^{n-1} B_{0}
\end{array}\right] B_{0}\right]=m
$$ 


\section{CONCLUSIONS}

This paper has considered discrete linear repetitive processes in the presence of the most general set of boundary conditions, i.e., where the state initial vector on each pass is an explicit function of the previous pass profile (output) vector. It has been shown, by the development of necessary and sufficient conditions, that the stability of these processes is completely determined by the structure of the pass initial state vector sequence. In particular, if this sequence is incorrectly modeled then incorrect stability conclusions will be reached.

Previous work has reported an equivalent 1-D linear systems state-space model of the dynamics of these processes. Unlike the 1-D models reported previously for other classes of 2-D linear systems, this 1-D model interpretation of the dynamics of discrete linear repetitive processes is defined by matrices and vectors of constant dimensions and the resulting system state-space matrices have constant entries. Here this 1-D equivalent model has been used to develop a complete characterization of the systems theoretic property of pass controllability for these processes in the form of conditions which can be checked by direct application of well known 1-D linear systems techniques. This characterization is not possible via any other route and strongly suggests that there exist other major roles for the equivalent 1-D state-space model interpretation of the dynamics of discrete linear repetitive processes. Here it has also been used to characterize so-called pass state controllability.

The final part of this paper has produced the first results on the important problem of stabilization by suitable choice of the pass state initial vector sequence. This again has made significant use of 1-D discrete linear systems results and, in particular, existence conditions for stability of such 1-D systems under state feedback.

\section{REFERENCES}

[1] N. Amann, D. H. Owens, and E. Rogers, "Iterative learning control using optimal feedback and feedforward actions," Int. J. Control, vol. 65, no. 2, pp. 277-293, 1996.

[2] - "Predictive optimal iterative learning control," Int. J. Control, vol. 69 , no. 2, pp. 203-226, 1998

[3] J. L. Aravena, M. Shafiee, and W. A. Porter, "State models and stability for 2-D filters," IEEE Trans. Circuits Syst., vol. 37, pp. 1509-1519, Dec. 1990.

[4] J. B. Edwards, "Stability problems in the control of multipass processes," Proc. Inst. of Electr. Eng., vol. 121, no. 11, pp. 1425-1431, 1974.

[5] E. Fornasini and G. Marchesini, "Doubly-indexed dynamical systems: State space models and structural properties," Math. Syst. Theory, vol. 12, pp. 59-72, 1978.

[6] K. Galkowski, E. Rogers, and D. H. Owens, "Matrix rank based conditions for reachability/controlability of discrete linear repetitive processes," Linear Algebra Appl., pp. 275-276, 1998.

[7] K. Galkowski, E. Rogers, A. Gramacki, J. Gramacki, and D. H. Owens, "Stability and dynamic boundary condition decoupling analysis for a class of 2-D discrete linear systems," Proc. Inst. of Electr. Eng. Circuits Devices Syst, vol. 148, no. 3, pp. 126-134, 2001.

[8] P. D. Roberts, "Numerical investigation of a stability theorem arising from the 2-dimensional analysis of an iterative optimal control algorithm," Multidimensional Syst. Signal Process., vol. 11, no. 1/2, pp. 109-124, 2000.

[9] R. P. Roesser, "A discrete state space model for linear image processing," IEEE Trans Automat Control, vol. AC-20, pp. 1-10, Jan. 1975.

[10] E. Rogers and D. H. Owens, "Stability Analysis for Linear Repetitive Processes," in Lecture Notes in Control and Information Sciences Series. Berlin, Germany: Springer Verlag, 1992, vol. 175.

[11] K. J. Smyth, "Computer Aided Analysis for Linear Repetitive Processes," PhD, Univ. of Strathclyde, Glasgow, U.K, 1992.

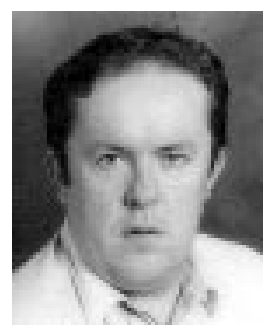

E. Rogers was born in 1956 in Northern Ireland. He received the undergraduate degree in mechanical engineering from The Queen's University, Belfast, U.K., and was the Ph.D. degree in multidimensional systems theory from The University of Sheffield, Sheffield, U.K.

He has been with The University of Southampton, Southampton, U.K. since 1990, where he is currently Professor of Control Systems Theory and Design in The Department of Electronics and Computer Science. Prior to moving to Southampton, he held lectureship posts in The Queen's University, (1984-1987) and the University of Strathclyde, Glasgow, U.K. (1988-1990). His current major research interests include multidimensional systems theory and applications, with particular emphasis on behavioral systems theory approaches and systems with repetitive dynamics, iterative learning control, flow control, and active control of microvibrations.

Dr. Rogers is the Editor of The International Journal of Control, an Associate Editor of Multidimensional Systems and Signal Processing, and Joint Editor of The Taylor and Francis research book series on Systems and Control. In addition, he has served extensively on IEEE, IFAC and IEE technical committees and acted as a consultant to numerous companies and government agencies in the U.K. and abroad.

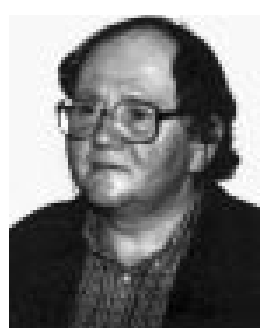

K. Galkowski received the M.S., Ph.D., and Habilitation degrees in electronics / automatic control from Technical University of Wrocaw, Wrocaw, Poland, in 1972, 1977, and 1994, respectively.

In October 1996 he joined the Technical University of Zielona Góra (now the University of Zielona Góra), Poland where he holds the Professor position. Recently, he has been awarded by the Visiting Professor position in the University of Southampton, Southampton, U.K. His research interests include multivariable $(n D)$ systems, repetitve processestheory and applications, control and related numerical and symbolic algebra methods. He is an author/editor of three monographs /books and over 80 research texts published in the leading journals and proceedings of international conferences.

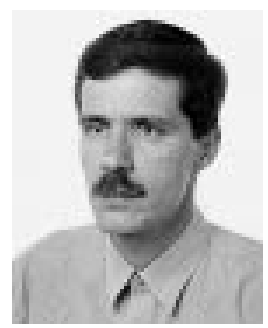

A. Gramacki received the diploma in electrical engineering, and the Ph.D. degree from the Technical University of Zielona Gora, Zielona Gora, Poland, in 1991 and 2000, respectively.

He was involved with Geographical Information Systems, and Data Management in the information technology industry, from 1991 to 1996 . He is currently an Assistant Professor at the same university (now called the University of Zielona Gora) since August 2001. His research interests are in the area of multidimensional $(n D)$ systems, scientific computing, numerical methods and particular, their application in systems and control problems of repititive processes. He is also active in the area of data processing and management through his teaching and professional activities.

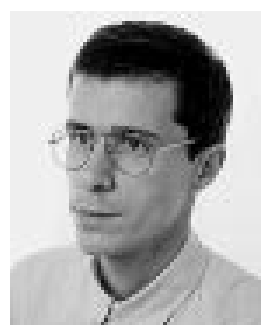

J. Gramacki received the diploma in electrical engineering, and the Ph.D. degree from the Technical University of Zielona Gora, Zielona Gora, Poland, in 1991 and 2000, respectively.

He was involved with Geographical Information Systems, and Data Management in the information technology industry, from 1991 to 1996 . He is currently an Assistant Professor at the same university (now called the University of Zielona Gora) since August 2001. His research interests are in the area of multidimensional $(n D)$ systems, scientific computing, numerical methods and particular, their application in systems and control problems of repititive processes. He is also active in the area of data processing and management through his teaching and professional activities. 


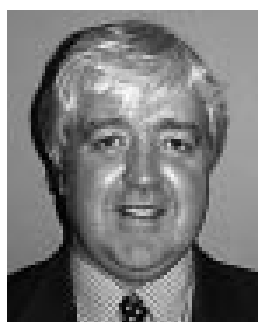

D. H. Owens is currently Head of Department of Automatic Control and Systems Engineering at the University of Sheffield, Sheffield, U.K. He has previously been Professor of Engineering Mathematics and Mechanical Engineering at the University of Strathclyde, Glasgow, U.K. (1985-1990) and the Professor and Head of the School of Engineering and Computer Science, at the University of Exeter, U.K. (1990-1999). His research interest include the theoretical aspects of systems modeling, controller design, and control algorithm development and has experience of applications in the nuclear industry, coal-fired power plant, building systems control and pneumatic conveying systems. He has several research grants, three textbooks and over 380 publications to his credit. He is currently a member of the IEE Council and the ImechE Machine Systems, Computing and Control Committee, the Chairman of the United Kingdom Automatic Control Council for 1999 through 2002, and is an independent member of the U.K. Health and Safety Executive Nuclear Safely Committee. 